\title{
STABLE EXPANSIONS IN HOMOGENEOUS POLYNOMIALS
}

\author{
LEV AIZENBERG AND NIKOLAI TARKHANOV
}

(Communicated by XXX)

\begin{abstract}
An expansion for a class of functions is called stable if the partial sums are bounded uniformly in the class. Stable expansions are of key importance in numerical analysis where functions are given up to certain error. We show that expansions in homogeneous functions are always stable on a small ball around the origin, and evaluate the radius of the largest ball with this property.
\end{abstract}

\section{Contents}

Introduction

1. Trigonometric series

2. A theorem of Rogosinski

3. Stable expansions

4. Eigenfunction expansions

5. Spherical harmonics

6. Lebesgue constants

7. Power series

8. Landau constants in higher dimensions

References

\section{INTRODUCTION}

In $1914 \mathrm{H}$. Bohr proved that there is an $r \in(0,1)$ such that if a power series converges in the unit disk $\mathbb{D}$ and its sum has modulus less than 1 then, for $|z|<r$, the sum of absolute values of its terms is again less than 1 . As was shown later by M. Riesz, I. Shur and F. Wiener, one can take $r=1 / 3$ and this constant cannot be improved.

We have not been aware of any essential motivation for Bohr's result but a stable convergence of power series which is of great importance for computations with power series. Namely, if a power series converges in the unit disk to a bounded function $f$ then its partial sums satisfy $\left|S_{N} f(z)\right| \leq\|f\|_{L^{\infty}(\mathbb{D})}$ for all $|z|<r$ and $N=0,1, \ldots$

Received by the editors August 31, 2005

2000 Mathematics Subject Classification. Primary 42C15; Secondary 41A44, 32A05.

Key words and phrases. Power series expansions, Fourier series, spherical harmonics, Lebesgue constants.

(C)2000 American Mathematical Society 
The theorem of $\mathrm{H}$. Bohr is merely intermediate in the verification of the last assertion. There is a direct proof of the stable convergence result due to Rogosinski [27] which actually shows that the constant $r=1 / 3$ can be enlarged to $r=1 / 2$ in the new setting.

Moreover, when focused on stable convergence, one naturally requires an estimate

$$
\left|S_{N} f(z)\right| \leq C\|f\|_{L^{\infty}(\mathbb{D})}
$$

for all $|z|<r$ and $N=0,1, \ldots$, with $C$ a constant independent of $N$ and $f$. As but one consequence of what is shown in this paper we mention that for any $r \in(0,1)$ there is a constant $C \geq 1$ depending on $r$, such that (0.1) holds for all $|z|<r$ and $N=0,1, \ldots$.

Each bounded holomorphic function $f$ in $\mathbb{D}$ is known to have weak limit values on the unit circle $\mathbb{S}$ which thus belong to the Hardy space $H^{\infty}(\mathbb{D})$. When restricted to $\mathbb{S}$, the power series expansion of $f$ becomes a Fourier series over the orthonormal system $\left\{e^{\imath k}\right\}_{k=0}^{\infty}$ of eigenfunctions of the Laplace-Beltrami operator on $\mathbb{S}$. The estimate $(0.1)$ is no longer valid for $|z|=1$, the minimal constant $C=C(N)$ behaves like

$$
C(N)=\frac{1}{\pi} \log N+O(1)
$$

for $N \rightarrow \infty$.

The constant (0.2) is called the Landau constant and it just amounts to the norm of the operator $f \rightarrow S_{N} f$ acting in the Hardy space $H^{\infty}(\mathbb{D})$.

For general Fourier series over the orthonormal system $\left\{e^{\imath k t}\right\}_{k=-\infty}^{\infty}$ the norm of the operator $f \rightarrow S_{N} f$ acting on all of the Banach space $L^{\infty}(\mathbb{S})$ is of crucial importance. This constant is usually referred to as Lebesgue constant, cf. [26, 8, 9, $10]$. It bears an asymptotic similar to $(0.2)$, the only difference being in replacing the factor $1 / \pi$ by larger $4 / \pi^{2}$.

We have thus specified the problem treated by Rogosinski [27] within the Fourier analysis on manifolds. It has been evolved in the far-reaching modern theory of eigenfunction expansions for selfadjoint elliptic operators or boundary value problems, cf. [28, 29] and references therein. With this as our starting point, we examine in this paper stable expansions in homogeneous functions. Our basic example is the expansion in spherical harmonics which goes as far as Mehler [22], cf. also $[23,25,7,11,21]$.

In $\S 1$ we start with trigonometric series on the unit circle and extend them to power series expansions for harmonic functions in the unit disk. We show how the analysis of Fourier series on the circle implies that power series expansions for harmonic functions in a disk are stable in any concentric disk of smaller radius.

In $\S 2$ we give a proof of the theorem of Rogosinski in the context of harmonic functions. In contrast to the original proof of [27] it is based on properties of the Fejer kernel.

In $\S 3$ we discuss abstract expansions in homogeneous functions in a domain, as they arise in the theory of partial differential equations. They fail in general to be related to any orthogonal expansions on the domain boundary. We show conditions of stability.

In $\S 4$ we restrict our attention to expansions in eigenfunctions of a selfadjoint elliptic differential operator on a compact Riemannian manifold. These are direct 
generalisations of trigonometric Fourier series, and they need not converge uniformly for continuous functions. To sum up such expansions one uses the so-called Riesz means $R_{N}^{\delta} f$ of order $\delta \geq 0$. We recall sharp asymptotic estimates for Riesz means in the case of second order elliptic operators. When operating in the space of bounded functions, the Riesz means are bounded uniformly in $N$, provided that their order is large enough.

In $\S 5$ we study expansions in spherical harmonics and highlight the nature of zonal harmonics.

In $\S 6$ we compute Lebesgue constants for spherical harmonic expansions and find explicit values of $r$ depending on the order of Riesz means.

In $\S 7$ we restrict our attention to power series expansions for separately harmonic functions in Reinhardt domains in $\mathbb{C}^{n}$. Such functions are closely related to holomorphic functions, and we explicitly compute the Rogosinski radius for simple bases in the space.

We finish the paper by discussing in $\S 8$ Landau constants, which are substitutes for Lebesgue constants in the case of power series expansions for holomorphic functions.

\section{Trigonometric SERIES}

Recall that the system of exponential functions $\left\{e^{\imath k t}\right\}_{k=-\infty}^{\infty}$ is a complete orthonormal system in the space $L^{2}(\mathbb{S})$ equipped with the scalar product

$$
(f, g)=\frac{1}{2 \pi} \int_{\mathbb{S}} f(y) \overline{g(y)} d s(y)
$$

$d s$ being a length form on $\mathbb{S}$. Any function $f \in L^{2}(\mathbb{S})$ possesses a Fourier series expansion

$$
f\left(e^{\imath t}\right)=\sum_{k=-\infty}^{\infty} c_{k} e^{\imath k t}
$$

converging in the $L^{2}(\mathbb{S})$-norm to $f$, where the coefficients are given by $c_{k}=\left(f, e^{\imath k s}\right)$ for $k \in \mathbb{Z}$.

Denote by $f(z)=f\left(r e^{\imath t}\right)$ the harmonic continuation of $f$ into $\mathbb{D}$ by the Poisson integral. In this way we obtain a function of Hardy class $H^{2}(\mathbb{D})$ which expands in homogeneous harmonic polynomials

$$
f(z)=\sum_{k=-\infty}^{-1} c_{k} \bar{z}^{-k}+\sum_{k=0}^{\infty} c_{k} z^{k}
$$

in all of $\mathbb{D}$, the series converging uniformly in each disk of radius less than 1 with center at the origin.

Theorem 1.1. The expansion (1.2) is stable.

Proof. Let $f$ be a bounded harmonic function in $\mathbb{D}$. Then $f$ has weak limit values on the circle $\mathbb{S}$ belonging to $L^{\infty}(\mathbb{S})$. 
The limit function $f\left(e^{\imath t}\right)$ expands as a Fourier series $(1.1)$ converging in $L^{2}(\mathbb{S})$. We have

$$
\begin{aligned}
S_{N} f\left(e^{\imath t}\right) & =\sum_{k=-N}^{N} c_{k} e^{\imath k t} \\
& =\frac{1}{2 \pi} \int_{-\pi}^{\pi} D_{N}(t-s) f\left(e^{\imath s}\right) d s
\end{aligned}
$$

for all $t \in[-\pi, \pi]$, where

$$
\begin{aligned}
D_{N}(t-s) & =\sum_{k=-N}^{N} e^{\imath k t} e^{-\imath k s} \\
& =\frac{\sin (N+1 / 2)(t-s)}{\sin 1 / 2(t-s)}
\end{aligned}
$$

is called the Dirichlet kernel, cf. [15, 5.1]. Hence it follows that the norm of the operator $S_{N}$ acting in $L^{\infty}(\mathbb{S})$ just amounts to

$$
\begin{aligned}
\left\|S_{N}\right\|_{\mathcal{L}\left(L^{\infty}(\mathbb{S})\right)} & =\frac{1}{2 \pi} \int_{-\pi}^{\pi}\left|D_{N}(s)\right| d s \\
& =\frac{4}{\pi^{2}} \log N+O(1)
\end{aligned}
$$

for $N \rightarrow \infty$, cf. (5.1.10) ibid.

The estimates (1.3) already allow one to prove the stability of the expansion $(1.2)$ on compact subsets of $\mathbb{D}$. To this end, write any $z \in \mathbb{D}$ in the form $z=r e^{\imath t}$ with $r \in[0,1)$ and $t \in[-\pi, \pi]$. Then

$$
\begin{aligned}
c_{-k} \bar{z}^{k}+c_{k} z^{k} & =r^{k}\left(c_{-k} e^{-\imath k t}+c_{k} e^{\imath k t}\right) \\
& =r^{k}\left(S_{k} f\left(e^{\imath t}\right)-S_{k-1} f\left(e^{\imath t}\right)\right)
\end{aligned}
$$

holds for each $k=1,2, \ldots$, whence

$$
\begin{aligned}
S_{N} f(z) & =\sum_{k=0}^{N} r^{k}\left(S_{k} f\left(e^{\imath t}\right)-S_{k-1} f\left(e^{\imath t}\right)\right) \\
& =(1-r) \sum_{k=0}^{N-1} r^{k} S_{k} f\left(e^{\imath t}\right)+r^{N} S_{N} f\left(e^{\imath t}\right) .
\end{aligned}
$$

As mentioned, the limit function $f\left(e^{\imath t}\right)$ on the circle is bounded. This readily implies

$$
\begin{aligned}
\left|S_{N} f(z)\right| & \leq \sup _{N=0,1, \ldots}\left((1-r) \sum_{k=0}^{N-1} r^{k}\left\|S_{k}\right\|_{\mathcal{L}\left(L^{\infty}(\mathbb{S})\right)}+r^{N}\left\|S_{N}\right\|_{\mathcal{L}\left(L^{\infty}(\mathbb{S})\right)}\right)\|f\|_{L^{\infty}(\mathbb{S})} \\
(1.5) & =C\|f\|_{L^{\infty}(\mathbb{S})}
\end{aligned}
$$

whenever $|z| \leq r$, which is due to the maximum principle. To complete the proof, it is sufficient to observe that the constant $C$ depending on $r$ is finite for all $r<1$ by (1.3), as desired.

Note that for any fixed $N=1,2, \ldots$ and $r \in(0,1)$ the expression under the supremum sign in (1.5) is a strongly increasing function of either variable $\left\|S_{1}\right\|_{\mathcal{L}\left(L^{\infty}(\mathbb{S})\right)}, \ldots,\left\|S_{N}\right\|_{\mathcal{L}\left(L^{\infty}(\mathbb{S})\right)}$. It just amounts to 1 if all the variables are equal 
to 1 . Hence, (1.3) shows that the constant $C$ in (1.5) is actually larger than 1 for all $r \in(0,1)$.

\section{A theOREM OF Rogosinski}

Theorem 2.1. Let (1.2) converge for each $|z|<1$ and $|f(z)| \leq 1$. Then the modulus of

$$
S_{N} f(z):=\sum_{k=-N}^{-1} c_{k} \bar{z}^{-k}+\sum_{k=0}^{N} c_{k} z^{k}
$$

does not exceed 1 for all $|z| \leq 1 / 2$ and $N=0,1, \ldots$

Proof. To derive the explicit estimates of Theorem 2.1 we make use of refined estimates of partial sums $S_{N} f(z)$ through the Cesàro means $\Sigma_{k} f\left(e^{\imath t}\right)$. These latter are defined by

$$
\begin{aligned}
\Sigma_{N} f\left(e^{\imath t}\right) & =\frac{1}{N+1}\left(S_{0} f\left(e^{\imath t}\right)+\ldots+S_{N} f\left(e^{\imath t}\right)\right) \\
& =\sum_{k=-N}^{N}\left(1-\frac{|k|}{N+1}\right) c_{k} e^{\imath k t}
\end{aligned}
$$

for $t \in[-\pi, \pi]$. It is easy to verify that

$$
\Sigma_{N} f\left(e^{\imath t}\right)=\frac{1}{2 \pi} \int_{-\pi}^{\pi} F_{N}(t-s) f\left(e^{\imath s}\right) d s
$$

where

$$
\begin{aligned}
F_{N}(t-s) & =\sum_{k=-N}^{N}\left(1-\frac{|k|}{N+1}\right) e^{\imath k(t-s)} \\
& =\frac{1}{N+1}\left(\frac{\sin 1 / 2(N+1)(t-s)}{\sin 1 / 2(t-s)}\right)^{2}
\end{aligned}
$$

is called the Fejer kernel, cf. [15, 5.1]. Hence we deduce that the norm of the operator $\Sigma_{N}$ acting in $L^{\infty}(\mathbb{S})$ is equal to

$$
\begin{aligned}
\left\|\Sigma_{N}\right\|_{\mathcal{L}\left(L^{\infty}(\mathbb{S})\right)} & =\frac{1}{2 \pi} \int_{-\pi}^{\pi}\left|F_{N}(s)\right| d s \\
& =1
\end{aligned}
$$

for all $N=0,1, \ldots$, cf. (5.1.8) ibid.

Pick $z \in \mathbb{D}$. Write $z=r e^{\imath t}$, where $r \in[0,1)$ and $t \in[-\pi, \pi]$. Once again using (1.4) we get

$$
\begin{aligned}
S_{N} f(z) & =\sum_{k=0}^{N} r^{k}\left(\sum_{j=0}^{k} S_{j} f\left(e^{\imath t}\right)-2 \sum_{j=0}^{k-1} S_{j} f\left(e^{\imath t}\right)+\sum_{j=0}^{k-2} S_{j} f\left(e^{\imath t}\right)\right) \\
& =\sum_{k=0}^{N} r^{k}\left((k+1) \Sigma_{k} f\left(e^{\imath t}\right)-2 k \Sigma_{k-1} f\left(e^{\imath t}\right)+(k-1) \Sigma_{k-2} f\left(e^{\imath t}\right)\right)
\end{aligned}
$$

which simplifies to

$$
(1-r)^{2} \sum_{k=0}^{N-2} r^{k}(k+1) \Sigma_{k} f\left(e^{\imath t}\right)+(1-2 r) r^{N-1} N \Sigma_{N-1} f\left(e^{\imath t}\right)+r^{N}(N+1) \Sigma_{N} f\left(e^{\imath t}\right) .
$$


Since $\left|f\left(e^{\imath t}\right)\right| \leq 1$ for all $t \in[-\pi, \pi]$, we apply (2.2) to see that $\left|\Sigma_{k} f\left(e^{\imath t}\right)\right| \leq 1$ for all $k=0,1, \ldots$ It follows that

$$
\begin{aligned}
\left|S_{N} f(z)\right| & \leq(1-r)^{2}\left(\frac{1-r^{N}}{1-r}\right)^{\prime}+|1-2 r| r^{N-1} N+r^{N}(N+1) \\
& =1+(|1-2 r|-(1-2 r)) r^{N-1} N
\end{aligned}
$$

whenever $|z| \leq r$, which is a consequence of the maximum principle for harmonic functions.

If $r \leq 1 / 2$ then (2.3) gives $\left|S_{N} f(z)\right| \leq 1$ for all $|z| \leq r$, which completes the proof.

Note that for $1 / 2<r<1$ the inequality (2.3) yields $\left|S_{N} f(z)\right| \leq C$ for $|z| \leq r$, where

$$
C=\sup _{N=0,1, \ldots}\left(1+2(2 r-1) r^{N-1} N\right)
$$

This proves again the stability of expansion (1.2), however, with a worse constant $C$ than that given by (1.5).

Another way of stating Theorem 2.1 is to say that if $f$ is a bounded harmonic function in $\mathbb{D}$ then

for all $|z| \leq 1 / 2$ and $N=0,1, \ldots$.

$$
\left|S_{N} f(z)\right| \leq\|f\|_{L^{\infty}(\mathbb{D})}
$$

For power series expansions of holomorphic functions in the disk Theorem 2.1 is due to Rogosinski [27]. He also proved that the constant $r=1 / 2$ cannot be improved, cf. $\S 7$. Since holomorphic functions are harmonic, the constant $r=1 / 2$ in Theorem 2.1 cannot be improved, too. In this way we obtain what Aizenberg et al. [4] call the Rogosinski radius.

\section{Stable Expansions}

Let $\mathbb{B}=\left\{z \in \mathbb{R}^{n}:|z|<1\right\}$ be the unit ball in $\mathbb{R}^{n}$, the boundary of $\mathbb{B}$ being the unit sphere $\mathbb{S}^{n-1}$ in $\mathbb{R}^{n}$.

Assume that $A$ is an elliptic homogeneous differential operator of order $m$ with constant coefficients in a neighbourhood of the closure of $\mathbb{B}$. Then there are diverse systems $\left\{P_{\alpha}\right\}_{\alpha \in \mathbb{Z}_{+}}$of homogeneous polynomials on $\mathbb{R}^{n}$ which form a basis in the space of solutions of $A f=0$ in $\mathbb{B}$, cf. [31, 2.3]. Here, $P_{\alpha}$ is of order $|\alpha|$. If $f$ is a solution to $A f=0$ in $\mathbb{B}$ of finite order of growth near $\mathbb{S}^{n-1}$ then $f$ can be expanded as

$$
f(z)=\sum_{\alpha \in \mathbb{Z}_{+}} c_{\alpha} P_{\alpha}(z)
$$

in a smaller ball with centre at the origin, the series converging uniformly. The coefficients are given by

$$
c_{\alpha}=-\int_{\mathbb{S}^{n-1}} G_{A}\left(D^{\alpha} \Phi(-y), f(y)\right)
$$

where $\Phi$ is the standard fundamental solution of convolution type for $A$, and $G_{A}$ a Green operator. This latter is a bidifferential operator of order $m-1$ which takes its values in differential forms of degree $n-1$. The integral over $\mathbb{S}^{n-1}$ is interpreted in a familiar way, for the derivatives of $f$ possess weak limit values on the boundary of $\mathbb{B}$. 
The partial sums $S_{N} f(z)$ of series (3.1) are well defined for all $z=x$ in $\mathbb{S}^{n-1}$. In fact,

$$
\begin{aligned}
S_{N} f(x) & :=\sum_{|\alpha| \leq N} c_{\alpha} P_{\alpha}(x) \\
& =-\int_{\mathbb{S}^{n-1}} G_{A}\left(K_{N}(x, y), f(y)\right)
\end{aligned}
$$

for $x \in \mathbb{S}^{n-1}$, where

$$
K_{N}(x, y)=\sum_{|\alpha| \leq N} P_{\alpha}(x) \otimes D^{\alpha} \Phi(-y) .
$$

Lemma 3.1. There are constants $a$ and $M$ depending only on $n$ and $A$, with the property that

$$
\left|S_{N} f(x)\right| \leq M a^{N}\|f\|_{W^{m-1, \infty}(\mathbb{B})}
$$

for all $x \in \mathbb{S}^{n-1}$ and $N=0,1, \ldots$

Proof. From the structure of a Green operator $G_{A}$ for $A$ it follows immediately that

$$
\left|S_{N} f(x)\right| \leq M^{\prime}\left(\sum_{|\alpha| \leq N}\left|P_{\alpha}(x)\right| \int_{\mathbb{S}^{n-1}} \sum_{|\beta| \leq m-1}\left|D^{\alpha+\beta} \Phi(-y)\right| d s(y)\right)\|f\|_{W^{m-1, \infty}(\mathbb{B})},
$$

with $M^{\prime}$ a constant depending only on $A$. In order to estimate the derivatives of the fundamental solution and the polynomials $P_{\alpha}$ on the unit sphere, we invoke Lemmas 2.2.3 and 2.3.3 of [31]. This makes it obvious that the sum in parentheses is majorised by $M^{\prime \prime} a^{N}$, where the constants $a$ and $M^{\prime \prime}$ are independent of $N$ and $f$, as desired.

This lemma shows that the norm of the operator $S_{N}$ acting from $W^{m-1, \infty}(\mathbb{B})$ to $L^{\infty}\left(\mathbb{S}^{n-1}\right)$ behaves like $M a^{N}$ for some $a>1$. The order of growth in $N$ differs qualitatively from that for partial sums of eigenfunction expansions on compact manifolds $S$ of dimension $n-1$, whose norms in $\mathcal{L}\left(L^{\infty}(S)\right)$ behave like $M N^{n-2 / 2}$, cf. $\S 4$.

Theorem 3.2. Expansion (3.1) is stable on a small ball about the origin in the sense that there are constants $r \in(0,1)$ and $C$ depending on $r$, with the property that

$$
\left|S_{N} f(z)\right| \leq C\|f\|_{W^{m-1, \infty}(\mathbb{B})}
$$

whenever $|z| \leq r$ and $f \in W^{m-1, \infty}(\mathbb{B})$.

Proof. Given any $z \in \mathbb{B}$, write $z=r x$ with $r \in[0,1)$ and $x \in \mathbb{S}^{n-1}$. Then for each $N=0,1, \ldots$ we get

$$
\begin{aligned}
S_{N} f(z) & =\sum_{|\alpha| \leq N} c_{\alpha} P_{\alpha}(r x) \\
& =\sum_{k=0}^{N} r^{k}\left(\sum_{|\alpha|=k} c_{\alpha} P_{\alpha}(x)\right) \\
& =\sum_{k=0}^{N} r^{k}\left(S_{k} f(x)-S_{k-1} f(x)\right)
\end{aligned}
$$


implying

$$
S_{N} f(z)=(1-r) \sum_{k=0}^{N-1} r^{k} S_{k} f(x)+r^{N} S_{N} f(x) .
$$

Using Lemma 3.1 now yields

$$
\begin{aligned}
\left|S_{N} f(z)\right| & \leq(1-r) \sum_{k=0}^{N-1} r^{k}\left|S_{k} f(x)\right|+r^{N}\left|S_{N} f(x)\right| \\
& \leq M\left((1-r) \sum_{k=0}^{N-1}(r a)^{k}+(r a)^{N}\right)\|f\|_{W^{m-1, \infty}(\mathbb{B})}
\end{aligned}
$$

which is obviously dominated by

$$
\frac{M}{1-r a}\|f\|_{W^{m-1, \infty}(\mathbb{B})}
$$

if $r<1 / a$. This completes the proof, for $M /(1-r a)$ is an increasing function of $r \in[0,1 / a)$.

\section{EIGENFUnCTION EXPANSIONS}

Suppose $S$ is a connected compact smooth manifold of dimension $n-1$ without boundary. Let $\Delta$ be a positive elliptic differential operator with smooth coefficients on $S$, selfadjoint with respect to a positive density $\mu$.

Let $\left\{\lambda_{k}\right\}_{k=0,1, \ldots}$ be the eigenvalues of $\Delta$ and $\left\{e_{k}\right\}_{k=0,1, \ldots}$ an orthonormal system of eigenfunctions, $e_{k}$ corresponding to $\lambda_{k}$. To every integrable function $f$ on $S$ we can associate a Fourier transform $\{\hat{f}(k)\}$ with respect to the system $\left\{e_{k}\right\}$, and an eigenfunction expansion

$$
f \simeq \sum_{k=0}^{\infty} \hat{f}(k) e_{k}
$$

Given any $\delta \geq 0$, the Riesz means of order $\delta$ of $f$ are defined by

$$
R_{N}^{\delta} f(x)=\sum_{\lambda_{k}<N+1}\left(1-\frac{\lambda_{k}}{N+1}\right)^{\delta} \hat{f}(k) e_{k}(x)
$$

for all $N=0,1, \ldots$. The behaviour of Riesz means, as $N \rightarrow \infty$, in various spaces $L^{p}(M, d \mu)$ for $1 \leq p \leq \infty$ has been extensively studied, cf. [6, 7, 28, 11, 12] and also the survey [5].

The family $R_{N}^{\delta} f$ obviously converges to $f$ in $L^{2}(M, d \mu)$ for all $\delta \geq 0$. However, $L^{p}$-convergence requires sufficiently large $\delta$. The optimal $\delta$ is known to be $\delta(p)=$ $(n-1)|1 / p-1 / 2|-1 / 2$, i.e., one could expect $L^{p}$-boundedness and convergence of $R_{N}^{\delta} f$ only for $\delta>\delta(p)$.

From negative results in $\mathbb{R}^{n-1}$ and a transplantation result in [19] it follows that, for small $\delta$, the family $R_{N}^{\delta} f$ does not necessarily converge in norm or pointwise to $f$, as $N \rightarrow \infty$, cf. also [21].

We are interested in the behaviour of Riesz means for bounded functions $f$, i.e., $p=\infty$ and $\delta(p)=n-2 / 2$. The bad behaviour of Riesz means for continuous or integrable functions has a natural measure in the Lebesgue constants, i.e., the 
integral norms of the Riesz kernels $K_{R_{N}^{\delta}}$. These latter generalise the Fejer kernel, namely,

$$
R_{N}^{\delta} f(x)=\int_{S} K_{R_{N}^{\delta}}(x, y) f(y) d \mu(y)
$$

for $x \in S$, where

$$
K_{R_{N}^{\delta}}(x, y)=\sum_{\lambda_{k}<N+1}\left(1-\frac{\lambda_{k}}{N+1}\right)^{\delta} e_{k}(x) \otimes \overline{e_{k}(y)} .
$$

As is known,

$$
\left\|R_{N}^{\delta}\right\|_{\mathcal{L}\left(L^{\infty}(S)\right)}=\sup _{x \in S} \int_{S}\left|K_{R_{N}^{\delta}}(x, y)\right| d \mu(y)
$$

which, given any fixed $N$, is obviously majorised by a decreasing function of $\delta \geq 0$. If $\delta \rightarrow \infty$ then

$$
\left\|R_{N}^{\delta}\right\|_{\mathcal{L}\left(L^{\infty}(S)\right)} \rightarrow \sup _{x \in S} \int_{S}\left|\sum_{\lambda_{k}=0} e_{k}(x) \otimes \overline{e_{k}(y)}\right| d \mu(y) .
$$

Example 4.1. If $\Delta$ is the Laplace-Beltrami operator on $S$ related to some Riemannian structure then the eigenspace of $\Delta$ corresponding to the eigenvalue $\lambda_{k}=0$ consists of all constant functions on $S$. Since the corresponding eigenfunction $e_{k}$ has norm 1 , we get

$$
\left\|R_{N}^{\delta}\right\|_{\mathcal{L}\left(L^{\infty}(S)\right)} \rightarrow \int_{S}\left|e_{k}\right|^{2} d \mu(y)
$$

as $\delta \rightarrow \infty$.

Sharp estimates for the norms of the operators $R_{N}^{\delta}$ in the cases of the classical Fourier series on the $(n-1)$-dimensional torus and on compact connected semisimple Lie groups were given in $[13,8,16,14]$. The paper [12] extends the estimates for the norms of Riesz means on $L^{\infty}(S)$ and its dual $L^{1}(S)$ to general eigenfunction expansions.

Theorem 4.2. Suppose $\Delta$ is of order 2. There exist two positive constants $M_{1}$ and $M_{2}$ such that, as $N \rightarrow \infty$,
1) $M_{1} N^{\frac{n-2}{2}-\delta} \leq\left\|R_{N}^{\delta}\right\|_{\mathcal{L}\left(L^{\infty}(S)\right)} \leq M_{2} N^{\frac{n-2}{2}-\delta}$ if $0 \leq \delta<(n-2) / 2$;
2) $\quad M_{1} \log N \leq\left\|R_{N}^{\delta}\right\|_{\mathcal{L}\left(L^{\infty}(S)\right)} \leq M_{2} \log N$ if $\delta=(n-2) / 2$;
3) $M_{1} \leq\left\|R_{N}^{\delta}\right\|_{\mathcal{L}\left(L^{\infty}(S)\right)} \leq M_{2}$ if $\delta>(n-2) / 2$.

Proof. Cf. [12].

Note that the estimate 3 ) is already in [28] for an arbitrary elliptic operator $\Delta$ of order $m$. In [29] some of these results are extended to the case of manifolds with boundary, where homogeneous Dirichlet boundary conditions are imposed on the eigenfunctions.

The knowledge of the order of increase of the operator norms of $R_{N}^{\delta}$ can be used to prove positive results on the convergence of Riesz means of functions in suitable subspaces of $L^{p}(S)$ or $C(S)$. See, e.g., [25, 5, 13, 10, 14]. 


\section{SPHERICAL HARMONICS}

Fourier analysis on manifolds is a generalisation of classical Fourier analysis, where one develops multi-periodic functions on $\mathbb{R}^{n}$ as series of exponentials and investigates the convergence properties of such developments. Multi-periodic functions can be regarded as functions on a torus, and the exponentials are eigenfunctions of the Laplace-Beltrami operator on the torus. On a general compact Riemannian manifold one may develop any reasonable function as a series in the eigenfunctions of the Laplace-Beltrami operator or any other selfadjoint elliptic operator and ask how well this series converges, depending on the regularity of the function.

Consider the unit sphere $\mathbb{S}^{n-1}$ in $\mathbb{R}^{n}$ equipped with normalised rotation-invariant measure $\mu$. For any $k \geq 0$, let $\mathcal{H}_{k}$ denote the space of all spherical harmonics of degree $k$ restricted to $\mathbb{S}^{n-1}$. As is known, $\mathcal{H}_{k}$ is the eigenspace of the LaplaceBeltrami operator on $\mathbb{S}^{n-1}$ corresponding to the eigenvalue $k(k+n-2)$, whose multiplicity is thus

$$
\sigma(n, k)=\left(\begin{array}{c}
n+k-1 \\
n-1
\end{array}\right)-\left(\begin{array}{c}
n+k-3 \\
n-1
\end{array}\right)
$$

if $k \geq 2$.

The space $L^{2}\left(\mathbb{S}^{n-1}\right)$ splits up into the orthogonal sum of $\mathcal{H}_{k}$. Moreover, every distribution $f$ on $\mathbb{S}^{n-1}$ has a spherical harmonic expansion

$$
f(x) \simeq \sum_{k=0}^{\infty} H_{k}(f)(x)
$$

for $x \in \mathbb{S}^{n-1}$, where $H_{k}(f) \in \mathcal{H}_{k}$. This is the expansion of $f$ in eigenfunctions of the Laplace-Beltrami operator on the sphere.

It is known [19] that if $1 \leq p<2$ then there is an $f \in L^{p}\left(\mathbb{S}^{n-1}\right)$ for which (5.1) diverges almost everywhere. That leaves open the general behaviour of spherical harmonic expansions for elements of $L^{2}\left(\mathbb{S}^{n-1}\right)$. For spherical harmonic expansions of zonal functions the problem is discussed in [21]. Recall that a function $f$ on $\mathbb{S}^{n-1}$ is said to be zonal about a point $y \in \mathbb{S}^{n-1}$ if $f(x)$ depends only on $\langle x, y\rangle$ for all $x \in \mathbb{S}^{n-1}$.

Recall that the Riesz means of order $\delta \geq 0$ of the expansion (5.1) are defined for each $N=0,1, \ldots$ by

$$
R_{N}^{\delta} f(x)=\sum_{k=0}^{N}\left(1-\frac{k}{N+1}\right)^{\delta} H_{k}(f)(x),
$$

where $x \in \mathbb{S}^{n-1}$. While being formally different from (4.2), the Riesz means (5.2) actually give an equivalent method of summability.

Theorem 21 of [17] tells us how the convergence of $R_{N}^{\delta} f(x)$ controls the size of the partial sums $S_{N} f(x)$.

Theorem 5.1. Suppose that $f$ is a distribution on the sphere for which there is some $\delta>0$ and $x \in \mathbb{S}^{n-1}$, such that $R_{N}^{\delta} f(x)$ converges to l as $N \rightarrow \infty$. Then for all $N$

$$
\left|S_{N} f(x)-l\right| \leq M N^{\delta} \sup _{k=0,1, \ldots, N+1}\left|R_{k}^{\delta} f(x)\right|
$$

with $M$ a constant depending only on $\delta$. 
This readily implies

$$
\begin{aligned}
H_{k}(f)(x) & =\left(S_{k}(f)(x)-l\right)-\left(S_{k-1}(f)(x)-l\right) \\
& =O\left(k^{\delta}\right),
\end{aligned}
$$

and so the individual terms of the spherical harmonic expansion have controlled growth, too.

In order to estimate the growth of the Lebesgue constants we must examine more closely the nature of the operator $R_{N}^{\delta}$. We start by considering the reproducing kernel for a finite dimensional subspace $\mathcal{H}_{k}$ of $L^{2}\left(\mathbb{S}^{n-1}\right)$. Thus, if $\left\{Y_{k, 1}, \ldots, Y_{k, \sigma(n, k)}\right\}$ is any orthonormal basis for $\mathcal{H}_{k}$, the orthogonal projection $H_{k}$ of $L^{2}\left(\mathbb{S}^{n-1}\right)$ onto $\mathcal{H}_{k}$ is given by

$$
H_{k}(f)(x)=\int_{\mathbb{S}^{n-1}} Z_{k}(x, y) f(y) d \mu(y)
$$

where

$$
Z_{k}(x, y)=\sum_{j=1}^{\sigma(n, k)} Y_{k, j}(x) \overline{Y_{k, j}(y)}
$$

are the so-called zonal harmonics.

The function $Z_{k}(x, y)$ is uniquely determined and is called the reproducing kernel for $\mathcal{H}_{k}$.

It follows that

$$
R_{N}^{\delta} f(x)=\int_{\mathbb{S}^{n-1}} K_{R_{N}^{\delta}}(x, y) f(y) d \mu(y)
$$

for $x \in S$, where

$$
K_{R_{N}^{\delta}}(x, y)=\sum_{k=0}^{N}\left(1-\frac{k}{N+1}\right)^{\delta} Z_{k}(x, y) .
$$

Thus, to compute the Lebesgue constants $\left\|R_{N}^{\delta}\right\|_{\mathcal{L}\left(L^{\infty}\left(\mathbb{S}^{n-1}\right)\right)}$, we must identify the kernel $K_{R_{N}^{\delta}}(x, y)$ and compute its $L^{1}$-norm.

In case $S=\mathbb{S}^{n-1}$ the homogeneity of $S$ under the action of the orthogonal group $G=\mathrm{O}(n)$ has been exploited to describe the reproducing kernel for $\mathcal{H}_{k}$ in terms of certain classical orthogonal polynomials, see e.g. [23, pp. 5-14]. This method gives precise growth estimates for $\left\|R_{N}^{\delta}\right\|_{\mathcal{L}\left(L^{\infty}\left(\mathbb{S}^{n-1}\right)\right)}$ in terms of known estimates on the $L^{1}$-norm of Jacobi polynomials.

First we notice that the spaces $\mathcal{P}_{N}$ and $\mathcal{H}_{k}$ are invariant under the mappings $f \mapsto g^{*} f$ for $g \in G$, where $g^{*} f(x)=f(g x)$. This is obvious for $\mathcal{P}_{N}$ since $x \mapsto g x$ is linear and thus whenever $p(x)$ is a polynomial of degree at most $N$, so is $p(g x)$. To see that $\mathcal{H}_{k}$ is invariant we observe that $f \mapsto g^{*} f$ is an orthogonal map of $L^{2}\left(\mathbb{S}^{n-1}\right)$ since $\mu$ is invariant under orthogonal maps of $\mathbb{R}^{n}$ which map $\mathbb{S}^{n-1}$ to itself. Thus $\mathcal{P}_{k-1}^{\perp}$ and hence the space $\mathcal{H}_{k}=\mathcal{P}_{k} \cap \mathcal{P}_{k-1}^{\perp}$ is invariant under the left translation by $G$.

Lemma 5.2. The kernel $Z_{k}(x, y)$ is a polynomial of degree at most $k$ in $x$ (and in y), such that
1) $Z_{k}(g x, g y)=Z_{k}(x, y)$ for all $g \in G$;
2) $\int_{\mathbb{S} n-1} Z_{k}(x, y) \overline{Z_{l}(x, y)} d \mu(y)=0$ for all $k \neq l$; 
3) $\int_{\mathbb{S}^{n-1}}\left|Z_{k}(x, y)\right|^{2} d \mu(y)=Z_{k}(x, x)$.

Proof. 1) follows from the uniqueness of the reproducing kernel for $\mathcal{H}_{k}$. And 2) and 3) are obvious.

The invariance property of Lemma 5.2 actually allows us to explicitly determine $Z_{k}(x, y)$.

\section{Lemma 5.3.}

1) Let $P_{k}(x, y)$ be a function on $\mathbb{S}^{n-1} \times \mathbb{S}^{n-1}$ which is a polynomial of degree at most $k$ in $x$ and in $y$. Suppose $P_{k}(g x, g y)=P_{k}(x, y)$ for every orthogonal map $g$. Then $P_{k}(x, y)=p_{k}(\langle x, y\rangle)$ for some polynomial $p_{k}(t)$ of degree $k$ on $\mathbb{R}$.

2) For each continuous function $f(t)$ on $[-1,1]$,

$$
\begin{array}{r}
\int_{\mathbb{S}^{n-1}} f(\langle x, y\rangle) d \mu(y)=1 / c \int_{-1}^{1} f(t)\left(1-t^{2}\right)^{\alpha} d t \\
\text { where } \alpha=\frac{n-3}{2} \text { and } c=\int_{-1}^{1}\left(1-t^{2}\right)^{\alpha} d t .
\end{array}
$$

Proof. 1) is a standard fact about invariants of the orthogonal group. Introduce an orthogonal basis $\left\{e_{1}, \ldots, e_{n}\right\}$ for $\mathbb{R}^{n}$. Then for any $x, y$ in $\mathbb{S}^{n-1}$ there exist orthogonal maps $g_{+}, g_{-}$such that $g_{ \pm} x=e_{1}$ and $g_{ \pm} y=\langle x, y\rangle e_{1} \pm \sqrt{1-\langle x, y\rangle^{2}} e_{2}$. Thus $P_{k}(x, y)=P_{k}\left(g_{ \pm} x, g_{ \pm} y\right)$ is a polynomial of degree $k$ in $\langle x, y\rangle$ and $\sqrt{1-\langle x, y\rangle^{2}}$ which is even in the latter variable. Hence it follows that $P_{k}(x, y)=p_{k}(\langle x, y\rangle)$, as desired.

2 ) is a standard fact which follows by introducing polar coordinates in $\mathbb{S}^{n-1}$ about $x$.

\section{Lebesgue Constants}

We can now identify the reproducing kernels on $\mathbb{S}^{n-1}$ and compute the Lebesgue constants.

Theorem 6.1. For $n \geq 2$,

$$
K_{R_{N}^{\delta}}(x, y)=\sum_{k=0}^{N}\left(1-\frac{k}{N+1}\right)^{\delta}\left(z_{k}^{(\alpha, \alpha)}\right)^{-1} P_{k}^{(\alpha, \alpha)}(1) P_{k}^{(\alpha, \alpha)}(\langle x, y\rangle)
$$

where $\alpha=(n-3) / 2, P_{k}^{(\alpha, \alpha)}$ is the Jacobi polynomial of degree $k$ and index $(\alpha, \alpha)$, and

$$
z_{k}^{(\alpha, \alpha)}=1 / c \int_{-1}^{1}\left|P_{k}^{(\alpha, \alpha)}(t)\right|^{2}\left(1-t^{2}\right)^{\alpha} d t
$$

with $c=\int_{-1}^{1}\left(1-t^{2}\right)^{\alpha} d t$.

Proof. We make use of formula (5.4) for $K_{R_{N}^{\delta}}(x, y)$ where $Z_{k}(x, y)$ is the kernel for the spherical harmonics of degree $k$. Now parts 1) of Lemmas 5.2 and 5.3 show that $Z_{k}(x, y)=z_{k}(\langle x, y\rangle)$ for some polynomials $z_{k}(t)$ of degree $k$. Parts 2$)$ of these lemmas show that

$$
\int_{-1}^{1} z_{k}(t) \overline{z_{l}(t)}\left(1-t^{2}\right)^{\alpha} d t=0
$$


for $k \neq l$, so $z_{k}(t)$ is a multiple of the Jacobi polynomial of degree $k$ and index $(\alpha, \alpha)$. Now the normalisation of Lemma $5.2,3)$ requires

$$
\begin{aligned}
z_{k}(1) & =z_{k}(\langle x, x\rangle) \\
& =1 / c \int_{-1}^{1}\left|z_{k}(t)\right|^{2}\left(1-t^{2}\right)^{\alpha} d t .
\end{aligned}
$$

This is uniquely satisfied by the multiple of the Jacobi polynomial

$$
z_{k}(t)=\left(z_{k}^{(\alpha, \alpha)}\right)^{-1} P_{k}^{(\alpha, \alpha)}(1) P_{k}^{(\alpha, \alpha)}(t),
$$

which yields the desired equality for $K_{R_{N}^{\delta}}(x, y)$.

Using Theorem 6.1 and part 2) of Lemma 5.3 we arrive at explicit expressions for Lebesgue constants

$$
\begin{aligned}
& \left\|R_{N}^{\delta}\right\|_{\mathcal{L}\left(L^{\infty}\left(\mathbb{S}^{n-1}\right)\right)} \\
& =\frac{1}{c} \int_{-1}^{1}\left|\sum_{k=0}^{N}\left(1-\frac{k}{N+1}\right)^{\delta}\left(z_{k}^{(\alpha, \alpha)}\right)^{-1} P_{k}^{(\alpha, \alpha)}(1) P_{k}^{(\alpha, \alpha)}(t)\right|\left(1-t^{2}\right)^{\alpha} d t
\end{aligned}
$$

for all $N=0,1, \ldots$ Combining this formula with results from Szegö [30] on Jacobi polynomials leads to estimates of the norm $\left\|R_{N}^{\delta}\right\|_{\mathcal{L}\left(L^{\infty}\left(\mathbb{S}^{n-1}\right)\right)}$ similar to those of Theorem 4.2, cf. [25, 11].

Once again we observe that for every fixed $N$ the norm $\left\|R_{N}^{\delta}\right\|_{\mathcal{L}\left(L^{\infty}\left(\mathbb{S}^{n-1}\right)\right)}$ tends to 1 when $\delta \rightarrow \infty$.

We are now in a position to prove an analogue of Theorem 2.1 in the context of spherical harmonic expansions. To this end, let $f$ be a harmonic function in the ball $B$ of finite order of growth near the boundary. Then $f$ has weak limit values on $\mathbb{S}^{n-1}$. It will cause no confusion if we use the same letter to designate the limit values. The distribution $f$ on the sphere can be expanded in spherical harmonics as (5.1), the series converges in the weak sense. Applying the Poisson formula to both sides of (5.1) we see that this series actually converges to the original harmonic function $f$ in the interior of $B$,

$$
f(z)=\sum_{k=0}^{\infty} H_{k}(f)(z)
$$

for all $|z|<1$, the convergence being uniform on any ball of radius less than 1 about the origin.

Theorem 6.2. The harmonic expansion (6.1) is stable on any compact subset of the ball B.

Proof. Let $f$ be a bounded harmonic function in $B$. Write any $z \in B$ in the form $z=r x$ with $r \in[0,1)$ and $x \in \mathbb{S}^{n-1}$. Then

$$
\begin{aligned}
H_{k}(f)(z) & =r^{k} H_{k}(f)(x) \\
& =r^{k}\left(S_{k} f(x)-S_{k-1} f(x)\right)
\end{aligned}
$$


holds for each $k=1,2, \ldots$, whence

$$
\begin{aligned}
S_{N} f(z) & =\sum_{k=0}^{N} r^{k}\left(S_{k} f(x)-S_{k-1} f(x)\right) \\
& =(1-r) \sum_{k=0}^{N-1} r^{k} S_{k} f(x)+r^{N} S_{N} f(x) .
\end{aligned}
$$

Obviously, the limit function $f(x)$ on the sphere is bounded. Hence it follows by Theorem 4.2 that

$$
\begin{aligned}
\left|S_{N} f(z)\right| & \leq M_{2} \sup _{N=0,1, \ldots}\left((1-r) \sum_{k=0}^{N-1} k^{\frac{n-2}{2}} r^{k}+N^{\frac{n-2}{2}} r^{N}\right)\|f\|_{L^{\infty}\left(\mathbb{S}^{n-1}\right)} \\
& =C\|f\|_{L^{\infty}\left(\mathbb{S}^{n-1}\right)}
\end{aligned}
$$

whenever $|z| \leq r$, which is due to the maximum principle. To complete the proof, it is sufficient to observe that the constant $C$ depending on $r$ is finite for all $r<1$, as desired.

Given any $\delta \geq 0$, we set

$$
L_{N}^{\delta}=\sup _{k=0,1, \ldots, N}\left\|R_{k}^{\delta}\right\|_{\mathcal{L}\left(L^{\infty}\left(\mathbb{S}^{n-1}\right)\right)}
$$

the suprema being bounded in $N$ provided that $\delta>(n-2) / 2$, which is due to Theorem 4.2 .

Theorem 6.3. Let (6.1) converge for each $|z|<1$ and $|f(z)| \leq 1$. Then the modulus of

$$
S_{N} f(z):=\sum_{k=0}^{N} H_{k}(f)(z)
$$

does not exceed $L_{N}^{\delta}$ for all $|z| \leq 1 / 2^{\delta}$ and $N=0,1, \ldots$

Proof. We make use of refined estimates of partial sums $S_{N} f(z)$ through the Riesz means $R_{k}^{\delta} f(x)$. Pick $z \in B$. Write $z=r x$, where $r \in[0,1)$ and $x \in \mathbb{S}^{n-1}$. An easy computation shows that

$$
\begin{aligned}
H_{0} & =R_{0}^{\delta}, \\
H_{1} & =2^{\delta} R_{1}^{\delta}-2^{\delta} R_{0}^{\delta}, \\
H_{2} & =3^{\delta} R_{2}^{\delta}-2^{\delta} 2^{\delta} R_{1}^{\delta}+\left(-3^{\delta}+2^{\delta} 2^{\delta}\right) R_{0}^{\delta}, \\
H_{3} & =4^{\delta} R_{3}^{\delta}-2^{\delta} 3^{\delta} R_{2}^{\delta}+\left(-3^{\delta}+2^{\delta} 2^{\delta}\right) 2^{\delta} R_{1}^{\delta}-\left(4^{\delta}-2^{\delta} 3^{\delta}-3^{\delta} 2^{\delta}+2^{\delta} 2^{\delta} 2^{\delta}\right) R_{0}^{\delta}, \\
H_{4} & =5^{\delta} R_{4}^{\delta}-2^{\delta} 4^{\delta} R_{3}^{\delta}+\left(-3^{\delta}+2^{\delta} 2^{\delta}\right) 3^{\delta} R_{2}^{\delta}-\left(4^{\delta}-3^{\delta} 2^{\delta}-2^{\delta} 3^{\delta}+2^{\delta} 2^{\delta} 2^{\delta}\right) 2^{\delta} R_{1}^{\delta} \\
& +\left(-5^{\delta}+2^{\delta} 4^{\delta}+3^{\delta} 3^{\delta}+4^{\delta} 2^{\delta}-2^{\delta} 2^{\delta} 3^{\delta}-3^{\delta} 2^{\delta} 2^{\delta}-2^{\delta} 3^{\delta} 2^{\delta}+2^{\delta} 2^{\delta} 2^{\delta} 2^{\delta}\right) R_{0}^{\delta},
\end{aligned}
$$

and so on, where we write $H_{k}$ instead of $H_{k}(f)(x)$ and $R_{k}^{\delta}$ instead of $R_{k}^{\delta} f(x)$ for short. We thus observe that

$$
H_{k}=\sum_{j=0}^{k}(-1)^{k-j} c_{k, j}(j+1)^{\delta} R_{j}^{\delta}
$$


for $k=0,1, \ldots$, where $c_{k, k}=1$ and

$$
\begin{aligned}
c_{k, j} & =c_{k-1, j-1}, \\
c_{k, 0} & =\sum_{j=1}^{k}(-1)^{j-1} c_{k, j}(j+1)^{\delta} .
\end{aligned}
$$

Since

$$
S_{N} f(z)=\sum_{k=0}^{N} r^{k} H_{k}(f)(x)
$$

it follows that

$$
\begin{aligned}
S_{0} f(z) & =R_{0}^{\delta}, \\
S_{1} f(z) & =\left(1-r 2^{\delta}\right) \sum_{k=0}^{0} r^{k}(k+1)^{\delta} R_{k}^{\delta}+r 2^{\delta} R_{1}^{\delta}, \\
S_{2} f(z) & =\left(1-r 2^{\delta}\right) \sum_{k=0}^{1} r^{k}(k+1)^{\delta} R_{k}^{\delta}+r^{2}\left(c_{2,0} R_{0}^{\delta}+3^{\delta} R_{2}^{\delta}\right), \\
S_{3} f(z) & =\left(1-r 2^{\delta}\right) \sum_{k=0}^{2} r^{k}(k+1)^{\delta} R_{k}^{\delta}+r^{2}\left(c_{2,0}-r c_{3,0}\right) \sum_{k=0}^{0} r^{k}(k+1)^{\delta} R_{k}^{\delta} \\
& +r^{3}\left(c_{3,1} 2^{\delta} R_{1}^{\delta}+4^{\delta} R_{3}^{\delta}\right), \\
S_{4} f(z) & =\left(1-r 2^{\delta}\right) \sum_{k=0}^{3} r^{k}(k+1)^{\delta} R_{k}^{\delta}+r^{2}\left(c_{2,0}-r c_{3,0}\right) \sum_{k=0}^{1} r^{k}(k+1)^{\delta} R_{k}^{\delta} \\
& +r^{4}\left(c_{4,0} R_{0}^{\delta}+c_{4,2} 3^{\delta} R_{2}^{\delta}+5^{\delta} R_{4}^{\delta}\right),
\end{aligned}
$$

and so on. In general, we get

$$
\begin{aligned}
S_{N} f(z) & =\left(1-r 2^{\delta}\right) \sum_{k=0}^{N-1} r^{k}(k+1)^{\delta} R_{k}^{\delta}+r^{2}\left(c_{2,0}-r c_{3,0}\right) \sum_{k=0}^{N-3} r^{k}(k+1)^{\delta} R_{k}^{\delta} \\
& +r^{4}\left(c_{4,0}-r c_{5,0}\right) \sum_{k=0}^{N-5} r^{k}(k+1)^{\delta} R_{k}^{\delta}+\ldots+r^{N} \sum_{N-k=\text { even }} c_{N, k}(k+1)^{\delta} R_{k}^{\delta} .
\end{aligned}
$$

Let us look more carefully at the partial sum $S_{1} f(z)$. Since $|f(x)| \leq 1$ for all $x \in \mathbb{S}^{n-1}$, we deduce that $\left|R_{k}^{\delta} f(x)\right| \leq L_{N}^{\delta}$ for every $k=0,1, \ldots, N$. It follows that

$$
\left|S_{1} f(z)\right| \leq\left(\left|1-r 2^{\delta}\right|+r 2^{\delta}\right) L_{1}^{\delta}
$$

whenever $|z| \leq r$, which is a consequence of the maximum principle for harmonic functions. It is easy to see that the expression in parentheses does not exceed 1 if and only if $r \leq 1 / 2^{\delta}$.

We now assume that $r=1 / 2^{\delta}$. Then for all $|z| \leq r$

$$
\left|S_{N} f(z)\right| \leq C \sup _{k=0,1, \ldots, N}\left\|R_{k}^{\delta}\right\|_{\mathcal{L}\left(L^{\infty}\left(\mathbb{S}^{n-1}\right)\right)}
$$


where

$$
\begin{aligned}
C & =r^{2}\left(c_{2,0}-r c_{3,0}\right) \sum_{k=0}^{N-3} r^{k}(k+1)^{\delta}+r^{4}\left(c_{4,0}-r c_{5,0}\right) \sum_{k=0}^{N-5} r^{k}(k+1)^{\delta}+\ldots \\
& +r^{N} \sum_{N-k=\text { even }} c_{N-k, 0}(k+1)^{\delta}
\end{aligned}
$$

just amounts to 1 , as desired.

As is seen from the proof, the constant $r=1 / 2^{\delta}$ seems to be sharp. To rigorously prove this, we need to show an extremal function. Namely, for each $z^{0} \in B$ satisfying $\left|z^{0}\right|>1 / 2^{\delta}$ there should exist a bounded harmonic function $f$ in the ball $B$, such that

$$
\left|S_{N} f\left(z^{0}\right)\right|>L_{N}^{\delta}\|f\|_{L^{\infty}(B)}
$$

for at least one number $N$. Note that $L_{N}^{\delta} \geq 1$, for $\left\|R_{0}^{\delta}\right\|_{\mathcal{L}\left(L^{\infty}\left(\mathbb{S}^{n-1}\right)\right)}$ is equal to 1 independently of $\delta$. Hence, our paper [3] gives an evidence to the contrary. Namely, the asymptotic of the Bohr radius for harmonic functions in a ball of $\mathbb{R}^{n}$, cf. [3], shows that an estimate (6.3) may hold for any $\left|z^{0}\right|>1 / 2^{\delta}$ only if $\log 2 / n \leq 1 / 2^{\delta}$. If for instance $\delta>(n-2) / 2$, then the domain of the latter inequality restricts to $n \leq 9$. Thus, [3] implies a stronger version of Theorem 6.3 if the dimension $n$ is large enough.

\section{POWER SERIES}

As mentioned in the Introduction, in the case of power series expansions the Lebesgue constants can be explicitly evaluated. This gives rise to introducing Bohr and Rogosinski radii, cf. [4], while no deep connections in mathematics has been observed yet.

Let $\mathcal{F}(D)$ be a space of continuous functions in a convex domain $D$ in $\mathbb{R}^{n}$ or $\mathbb{C}^{n}$, endowed with the topology of uniform convergence on compact subsets of $D$.

Suppose $\left\{f_{k}\right\}_{k=0}^{\infty}$ is a topological basis in $\mathcal{F}(D)$. By the Bohr radius for this basis is meant the largest number $r>0$ with the property that if $f \in \mathcal{F}(D)$ has an expansion

$$
f=\sum_{k=0}^{\infty} c_{k} f_{k}
$$

and satisfies the inequality $|f|<1$ in $D$ then

$$
\sum_{k=0}^{\infty}\left|c_{k} f_{k}\right|<1
$$

in the homothety $r D$. We denote the Bohr radius with $r_{B}$.

In a similar way one defines the Rogosinski radius for $\left\{f_{k}\right\}_{k=0}^{\infty}$, the only difference being in replacing (7.1) by the inequality

$$
\left|\sum_{k=0}^{N} c_{k} f_{k}\right|<1
$$

in the homothety $r D$ for all partial sums. The Rogosinski radius is denoted by $r_{R}$.

From (7.1) and (7.2) it follows readily that $r_{B} \leq r_{R}$ for all spaces $\mathcal{F}(D)$ and bases $\left\{f_{k}\right\}_{k=0}^{\infty}$. For the space of all holomorphic functions in the unit disk $\mathbb{D}$ and 
the basis $\left\{z^{k}\right\}_{k=0}^{\infty}$ a research of $\mathrm{H}$. Bohr (1914) resulted in $r_{B}=1 / 3$, cf. [20]. The classical result of Rogosinski [27] states that $r_{R}=1 / 2$ for the same space and the same basis.

There are many analogues of the phenomenon of Bohr in higher dimensions, cf. [1] and the references given there. It should be noted that no explicit value of the Bohr radius is still known for any space of holomorphic functions in a domain $D \subset \mathbb{C}^{n}$ with $n>1$. There are only certain estimates for this radius.

In [3] we first after Bohr indicated two cases where the Bohr radius can be explicitly computed. These are the space of harmonic functions on a ball in $\mathbb{R}^{n}$ and the space of separately harmonic functions in a polydisk in $\mathbb{C}^{n}$.

The paper [4] deals with analogues of Rogosinski radius in higher dimensions. In the case of a complete Reinhardt domain in $\mathbb{C}^{n}$ the Rogosinski radius is proved to be equal to $1 / 2$ for both partial sums

$$
\sum_{k_{1} \leq N_{1}, \ldots, k_{n} \leq N_{n}} c_{k} z^{k} \text { and } \sum_{k_{1}+\ldots+k_{n} \leq N} c_{k} z^{k}
$$

where $k=\left(k_{1}, \ldots, k_{n}\right)$ and $z^{k}=z_{1}^{k_{1}} \cdot \ldots \cdot z_{n}^{k_{n}}$.

Theorem 2.1 states that the Rogosinski radius for the basis $\left\{\bar{z}^{-k}, z^{k}\right\}_{k=0}^{\infty}$ in the space of complex-valued harmonic functions in the disk $\mathbb{D}$ is at least $1 / 2$. It is actually equal to $1 / 2$, for it does not exceed the Rogosinski radius for the basis $\left\{z^{k}\right\}_{k=0}^{\infty}$ in the space of holomorphic functions in $\mathbb{D}$. As extremal function in this latter case one can take

$$
f(z)=\frac{z-a}{1-\bar{a} z}
$$

where $|a|<1$. It is holomorphic in $\mathbb{D}$ and satisfies $|f(z)|<1$ for $|z|<1$. A trivial verification shows that $S_{1} f(z)=-a+\left(1-|a|^{2}\right) z$. Moreover, if

$$
\Re\left(-\frac{\bar{a}}{|a|} z\right)>\frac{1}{1+|a|}
$$

then $\left|S_{1} f(z)\right|>1$. Letting $|a| \rightarrow 1$ we deduce that the Rogosinski radius for the basis $\left\{z^{k}\right\}_{k=0}^{\infty}$ does not exceed $1 / 2$.

This result easily extends to separately harmonic functions in a Reinhardt domain $\mathcal{D} \subset \mathbb{C}^{n}$, i.e., functions which are harmonic in each complex variable $z_{1}, \ldots, z_{n}$. The space is endowed with the topology of uniform convergence on compact subsets of $\mathcal{D}$. As a basis in this space we can take the system $\left\{1, w_{I}^{\alpha}\right\}$ where $I$ varies over all $n$-tuples consisting of \pm 1 (there are $2^{n}$ such tuples), and $\alpha$ varies over all multi-indices in $\mathbb{Z}_{+}^{n}$. Write $I=\left(i_{1}, \ldots, i_{n}\right)$, then $w_{I}=\left(w_{I, 1}, \ldots, w_{I, n}\right)$ where $w_{I, j}=z_{j}$, if $i_{j}=+1$, and $w_{I, j}=\bar{z}_{j}$, if $i_{j}=-1$. Under this notation, we have $w_{I}^{\alpha}=w_{I, 1}^{\alpha_{1}} \ldots w_{I, n}^{\alpha_{n}}$, and any function $f(z, \bar{z})$ separately harmonic in $\mathcal{D}$ has a series expansion

$$
f(z, \bar{z})=\sum_{\alpha} \sum_{I} c_{\alpha, I} w_{I}^{\alpha}
$$

For every multi-index $N=\left(N_{1}, \ldots, N_{n}\right)$ in $\mathbb{Z}_{+}^{n}$, we introduce a partial sum of series (7.4) of the form

$$
S_{N} f(z, \bar{z})=\sum_{\alpha \leq N} \sum_{I} c_{\alpha, I} w_{I}^{\alpha}
$$


where the inequality $\alpha \leq N$ is understood component-wise, i.e., $\alpha_{j} \leq N_{j}$ for all $j=1, \ldots, n$.

Theorem 7.1. When defined with respect to partial sums (7.5), the Rogosinski radius for the basis $\left\{1, w_{I}^{\alpha}\right\}$ in the space of separately harmonic functions in the unit polydisk $\mathbb{D}^{n}$ is equal to $1 / 2$.

Proof. Denote the Rogosinski radius in question by $r_{R}$. Applying Theorem 2.1 in every variable $z_{j}, j=1, \ldots, n$, we deduce that $r_{R} \geq 1 / 2$. The equality $r_{R}=1 / 2$ now follows by considering each of the holomorphic functions $f\left(z_{j}\right)$ given by $(7.3)$, $j=1, \ldots, n$.

Since each complete Reinhardt domain is a union of polydisks, we arrive at the following consequence.

Corollary 7.2. The Rogosinski radius for the basis $\left\{1, w_{I}^{\alpha}\right\}$ in the space of separately harmonic functions in a complete Reinhardt domain $\mathcal{D} \subset \mathbb{C}^{n}$ is at least $1 / 2$.

Note that Theorem 2.1 is actually valid for any disk of finite radius in the plane. Combining this with Corollary 7.2, we conclude that if a complete Reinhardt domain $\mathcal{D} \subset \mathbb{C}^{n}$ is bounded with respect to at least one variable $z_{j}, j=1, \ldots, n$, then the Rogosinski radius for the basis $\left\{1, w_{I}^{\alpha}\right\}$ in the space of separately harmonic functions in $\mathcal{D}$ just amounts to $1 / 2$.

If a function $f(z, \bar{z})$ is expanded in a polydisk $\mathbb{D}_{r}^{n}=\left\{z:\left|z_{1}\right|<r_{1}, \ldots,\left|z_{n}\right|<r_{n}\right\}$ as a series (7.4), then the coefficients of this expansion are given by

$$
c_{\alpha, I}=\lim _{t \rightarrow 1-0} \frac{1}{(2 \pi)^{n}} \int_{s_{t r}} f(z, \bar{z}) \frac{1}{w_{I}^{\alpha}} \frac{d z_{1}}{z_{1}} \wedge \ldots \wedge \frac{d z_{n}}{z_{n}},
$$

where $s_{r}=\left\{z:\left|z_{1}\right|=r_{1}, \ldots,\left|z_{n}\right|=r_{n}\right\}$ stands for the $n$-dimensional skeleton of $\mathbb{D}_{r}^{n}$. Hence it follows that if $|f(z, \bar{z})| \leq M$ in the polydisk $\mathbb{D}_{r}^{n}$ then many-dimensional Cauchy inequalities $\left|c_{\alpha, I}\right| \leq M / r^{\alpha}$ are valid. Given any complete Reinhardt domain $\mathcal{D}$ in $\mathbb{C}^{n}$, one can apply the Cauchy inequalities to any polydisk lying in $\mathcal{D}$. In this way we obtain

Lemma 7.3. If $f(z, \bar{z})$ is expanded in a complete Reinhardt domain $\mathcal{D}$ as series (7.4) and $|f(z, \bar{z})| \leq M$ in $\mathcal{D}$, then

$$
\left|c_{\alpha, I}\right| \leq \frac{M}{\sup _{\mathcal{D}}\left|z^{\alpha}\right|} .
$$

The estimate (7.6) is a generalisation of an estimate, obtained in [2] for holomorphic functions and power series, to the case of separately harmonic functions and series expansions (7.4).

Corollary 7.4. If a bounded separately harmonic function $f(z, \bar{z})$ is expanded in the domain $\left\{z:\left|z_{1} \ldots z_{n}\right|<1\right\}$ as series (7.4), then this expansion contains only those monomials $w_{I}^{\alpha}$ which satisfy $\left|w_{I}^{\alpha}\right|=\left|z_{1} \ldots z_{n}\right|^{k}$ for some $k=0,1, \ldots$

Yet another class of harmonic functions closely related to holomorphic ones is constituted by pluriharmonic functions in a Reinhardt domain $\mathcal{D} \subset \mathbb{C}^{n}$. As usual, we give this space the topology of uniform convergence on compact subsets of $\mathcal{D}$. Pluriharmonic functions with real values are defined to be real parts of holomorphic functions, complex-valued pluriharmonic functions are obtained by tensoring with 
C. A simple basis in the space of pluriharmonic functions consists of the monomials $z^{\alpha}$ and $\bar{z}^{\alpha}$. The following theorem gives the Rogosinski radius of this basis with respect to partial sums (7.5).

Theorem 7.5. If a complete Reinhardt domain $\mathcal{D} \subset \mathbb{C}^{n}$ is bounded with respect to at least one variable $z_{j}, j=1, \ldots, n$, then the Rogosinski radius for the basis $\left\{z^{\alpha}, \bar{z}^{\alpha}\right\}$ in the space of pluriharmonic functions in $\mathcal{D}$ is equal to $1 / 2$.

Proof. This follows by the same method as in the case of separately harmonic functions.

If the Reinhardt domain $\mathcal{D}$ is not bounded with respect to any variable, then Theorem 7.5 fails in general.

Example 7.6. The Rogosinski radius for the basis $\left\{z^{\alpha}, \bar{z}^{\alpha}\right\}$ in the space of pluriharmonic functions in the domain $\mathcal{D}=\left\{z:\left|z_{1} \ldots z_{n}\right|<1\right\}$ is equal to $\sqrt[n]{1 / 2}$. Indeed, by Corollary 7.4 any pluriharmonic function $f(z, \bar{z})$ in $\mathcal{D}$ is expanded there as a series

$$
f(z, \bar{z})=\sum_{k=0}^{\infty} c_{-k}\left(\overline{z_{1} \ldots z_{n}}\right)^{k}+c_{k}\left(z_{1} \ldots z_{n}\right)^{k}
$$

i.e., is actually a harmonic function of one complex variable $z_{1} \cdot \ldots \cdot z_{n}$. It remains to apply Theorem 2.1 .

It is worth pointing out that harmonic functions in a ball in $\mathbb{R}^{n}, n>2$, are no longer closely related to holomorphic functions of complex variables. In contrast to Corollary 7.2, Theorem 6.3 puts us on to an idea that the Rogosinski radius for natural bases in the space of harmonic functions depends on $n$ and tends to 0 when $n \rightarrow \infty$.

\section{LANDAU CONSTANTS IN HighER DIMENSIONS}

Set

$$
L_{N}=\sup _{\|f\|_{L^{\infty}(\mathbb{D})} \leq 1} \max _{|z| \leq 1}\left|S_{N} f(z)\right|,
$$

where $S_{N} f$ is the $N$ th partial sum of the Taylor series

$$
f(z)=\sum_{k=0}^{\infty} c_{k} z^{k},
$$

and the supremum is over all holomorphic functions $f$ in the disk $\mathbb{D}$ whose modulus does not exceed 1.

Landau, cf. the book [20], showed a remarkable explicit equality for these constants, namely

$$
L_{N}=1+\left(\frac{1}{2}\right)^{2}+\left(\frac{1 \cdot 3}{2 \cdot 4}\right)^{2}+\ldots+\left(\frac{(2 N-1) ! !}{(2 N) ! !}\right)^{2}
$$

This makes it obvious that

for $N \rightarrow \infty$.

$$
L_{N} \sim \frac{\log N}{\pi}
$$

In this section we discuss an analogue of the Landau equality in the case of several variables. 
Let $f(z)$ be a holomorphic function in a complete Reinhardt domain $\mathcal{D} \subset \mathbb{C}^{n}$ and

$$
f(z)=\sum_{\alpha} c_{\alpha} z^{\alpha}
$$

in $\mathcal{D}$. There are two natural ways to define the partial sums of this power series, namely

$$
S_{N} f(z)=\sum_{\alpha \leq N} c_{\alpha} z^{\alpha}
$$

where $N$ is an $n$-tuple of non-negative integers and the inequality $\alpha \leq N$ is understood component-wise, and

$$
S_{N} f(z)=\sum_{|\alpha| \leq N} c_{\alpha} z^{\alpha}
$$

where $N$ is a non-negative integer and $|\alpha|=\alpha_{1}+\ldots+\alpha_{n}$ for a multi-index $\alpha$ in $\mathbb{Z}_{+}^{n}$. The latter partial sums are relevant not only for Reinhardt domains, where any holomorphic function expands as a power series, but also for complete Cartan domains, where any holomorphic function possesses an expansion in homogeneous polynomials

$$
f(z)=\sum_{k=0}^{\infty} P_{k}(z),
$$

$P_{k}$ being a homogeneous polynomial of degree $k$.

Theorem 8.1. Let $\mathcal{D}$ be a bounded complete Reinhardt domain in $\mathbb{C}^{n}$ and

$$
L_{N, \mathcal{D}}=\sup _{\|f\|_{L^{\infty}(\mathcal{D})} \leq 1} \max _{z \in \overline{\mathcal{D}}}\left|S_{N} f(z)\right|,
$$

for $N=\left(N_{1}, \ldots, N_{n}\right)$. Then

$$
L_{N, \mathcal{D}} \leq L_{N_{1}} \ldots L_{N_{n}}
$$

Proof. To show (8.3) it suffices to apply the result of Landau first in $z_{1}$, then in $z_{2}$, and so on.

If moreover $\mathcal{D}=\mathbb{D}^{n}$ is the unit polydisk then the inequality (8.3) actually turns into equality. To prove this, take the extremal functions $f_{N_{j}}\left(z_{j}\right)$ of Landau which satisfy

$$
\max _{z \in \overline{\mathbb{D}}}\left|S_{N_{j}} f_{N_{j}}(z)\right|=L_{N_{j}}
$$

cf. $[20, \S 2]$, and consider $f_{N}(z)=f_{N_{1}}\left(z_{1}\right) \ldots f_{N_{n}}\left(z_{n}\right)$. This is a holomorphic function in $\mathbb{D}^{n}$ satisfying $\left|f_{N}(z)\right| \leq 1$ and

$$
\max _{z \in \overline{\mathcal{D}}}\left|S_{N} f_{N}(z)\right|=L_{N_{1}} \ldots L_{N_{n}} .
$$

Combining this with $(8.3)$ we readily obtain the equality $L_{N, \mathcal{D}}=L_{N_{1}} \ldots L_{N_{n}}$, as desired.

We now consider a complete Cartan domain $\mathcal{D}$ in $\mathbb{C}^{n}$. Any section of $\mathcal{D}$ by a complex line $\left\{z: z_{j}=k_{j} t, t \in \mathbb{C}\right\}$ is a disk with centre at the origin. A Cartan domain $\mathcal{D}$ is said to be normal if almost all sections of $\mathcal{D}$ by complex lines through the origin are disks of finite radius. 
Theorem 8.2. Let $\mathcal{D}$ be a normal Cartan domain in $\mathbb{C}^{n}$ and

$$
L_{N, \mathcal{D}}=\sup _{\|f\|_{L}(\mathcal{D}) \leq 1} \max _{z \in \overline{\mathcal{D}}}\left|S_{N} f(z)\right|
$$

for $N=0,1, \ldots$, the partial sums being with respect to expansion (8.2). Then $L_{N, \mathcal{D}} \leq L_{N}$. If moreover $\mathcal{D}$ is bounded with respect to at least one complex variable then

$$
L_{N, \mathcal{D}}=L_{N}
$$

Proof. The inequality $L_{N, \mathcal{D}} \leq L_{N}$ follows immediately by applying the result of Landau to holomorphic functions of one complex variable in sections of $\mathcal{D}$ by complex lines through the origin. If the domain $\mathcal{D}$ is bounded with respect to a complex variable $z_{j_{0}}$, then for proving (8.4) it suffices to consider the extremal functions $f_{N}\left(z_{j_{0}}\right)$ of Landau, cf. [20, $\left.\S 2\right]$.

Let us mention an interesting consequence of Theorems 8.1 and 8.2 for a polydisk $\mathbb{D}_{r}^{n}$. Namely,

$$
\begin{aligned}
L_{(N, \ldots, N), \mathbb{D}_{r}^{n}} & \sim\left(\frac{\log N}{\pi}\right)^{n}, \\
L_{N, \mathbb{D}_{r}^{n}} & \sim \frac{\log N}{\pi}, \\
L_{n N, \mathbb{D}_{r}^{n}} & \sim \frac{\log n+\log N}{\pi}
\end{aligned}
$$

for $N \rightarrow \infty$. While the partial sums $S_{(N, \ldots, N)} f$ are squeezed by $S_{N} f$ from below and by $S_{n N} f$ from above, the second asymptotic is essentially different from the first and third asymptotics.

The condition that $\mathcal{D}$ is bounded with respect to at least one complex variable cannot be dropped in Theorem 8.2.

Example 8.3. As is shown in Example 7.6, any bounded holomorphic function in the domain $\mathcal{D}=\left\{z:\left|z_{1} \ldots z_{n}\right|<1\right\}$ depends actually on the single complex variable $z_{1} \ldots z_{n}$. Hence it follows that $L_{N, \mathcal{D}}=L_{[N / n]}$, where $[N / n]$ stands for the integer part of the number $N / n$.

In a number of theorems above we require that the Reinhardt domain $\mathcal{D}$ be complete. This condition can be weakened. Namely, it suffices to require that $\mathcal{D}$ would contain the origin. Any power series converging in such a domain $\mathcal{D}$ will automatically converge in a complete Reinhardt domain containing $\mathcal{D}$. Moreover, if $|f(z)| \leq 1$ for all $z \in \mathcal{D}$, then this inequality remains valid in the larger domain, too. 


\section{REFERENCES}

1. L. Aizenberg, Bohr theorem, In: Encyclopaedia of Math., Supplement II, Kluwer Academic Publishers, Dordrecht, NL, 2000.

2. L. Aizenberg and B. Mityagin, Spaces of analytic functions in multicircular domains, Siberian Math. J. 1 (1960), no. 2, 153-170.

3. L. Aizenberg and N. Tarkhanov, A Bohr phenomenon for elliptic equations, Proc. London Math. Soc. 82 (2001), 385-401.

4. L. Aizenberg, M. Elin, and D. Shoikhet, On the Rogosinski radius for holomorphic mappings and some its applications, Studia Mathematica 168 (2005), no. 2, 147-158.

5. S. A. Alimov, V. A. Il'in, and E. M. Nikishin, Convergence problems of multiple Fourier series and spectral decompositions. I, II, Russian Math. Surveys 31 (1976), 29-86; 32 (1977), $115-139$.

6. S. Bochner, Summation of multiple Fourier series by spherical means, Trans. Amer. Math. Soc. 40 (1936), 175-207.

7. A. Bonami and J.-L. Clerc, Sommes de Cesàro et multiplicateurs des développements en harmoniques sphériques, Trans. Amer. Math. Soc. 183 (1973), 223-263.

8. M. Carenini and P. M. Soardi, Sharp estimates for Lebesgue constants, Proc. Amer. Math. Soc. 89 (1983), no. 3, 449-452.

9. D. I. Cartwright, Lebesgue constants for Jacobi expansions, Proc. Amer. Math. Soc. 87 (1983), no. $3,427-433$

10. D. I. Cartwright and P. M. Soardi, Best conditions for the norm convergence of Fourier series, J. Approx. Theory 38 (1983), no. 4, 344-353.

11. L. Colzani, M. Taibleson, and G. Weiss, Maximal estimates for Cesàro and Riesz means on spheres, Indiana Univ. Math. J. 33 (1984), no. 6, 873-889.

12. L. Colzani and G. Travaglini, Estimates for Riesz kernels of eigenfunction expansions of elliptic differential operators on compact manifolds, J. Funct. Anal. 96 (1991), 1-30.

13. L. Colzani and P. M. Soardi, $L^{p}$ norms of certain kernels on the $n$-dimensional torus, Trans. Amer. Math. Soc. 266 (1981), 617-627.

14. B. Dreseler, On the asymptotic of the Bochner-Riesz kernel on compact Lie groups, J. Funct. Anal. 68 (1986), 374-387.

15. R. E. Edwards, Fourier Series, Vol. 1, Springer-Verlag, Berlin et al., 1979.

16. S. Giulini and G. Travaglini, Sharp estimates for Lebesgue constants on compact Lie groups, J. Funct. Anal. 68 (1986), 106-116.

17. G. H. Hardy and M. Riesz, The General Theory of Dirichlet's Series, Cambridge, 1915.

18. L. Hörmander, On the Riesz means of spectral functions and eigenfunction expansions for elliptic differential operators, In: Some Recent Advances in the Basic Sciences, Vol. 2, Yeshiva University, New York, 1969, 155-202.

19. C. Kenig, R. Stanton, and P. Tomas, Divergence of eigenfunction expansions, J. Funct. Anal. 46 (1982), 28-44.

20. E. Landau and D. Gaier, Darstellung und Begründung einiger neuerer Ergebniße der Funktionentheorie, Springer, Berlin et al., 1986.

21. Ch. Meaney, Divergent sums of spherical harmonics, In: International Conference on Harmonic Analysis and Related Topics (Sydney, 2002), Proc. Centre Math. Appl. Austral. Nat. Univ., 41, Canberra, 2003, 110-117.

22. F. G. Mehler, Ueber die Entwicklung einer Funktion von beliebig vielen Variablen nach Laplaceschen Funktionen höherer Ordnung, J. für die reine und angewandte Math. 66 (1866), 161-176.

23. C. Müller, Spherical harmonics, In: Lect. Notes Math., no. 17, Springer, Berlin et al., 1966.

24. D. L. Ragozin, Polynomial approximation on compact manifolds and homogeneous spaces, Trans. Amer. Math. Soc. 150 (1970), 41-53.

25. D. L. Ragozin, Uniform convergence of spherical harmonic expansions, Math. Ann. 195 (1972), 87-94.

26. H. Rau, Über die Lebesgueschen Konstanten der Reihenentwicklungen nach Jacobischen Polynomen, J. für die reine und angewandte Math. 161 (1933), 237-254.

27. W. Rogosinski, Über Bildschranken bei Potenzreihen und ihren Abschnitten, Math. Z. 17 (1923), 260-276. 
28. Ch. D. Sogge, On the convergence of Riesz means on compact manifolds, Ann. of Math. 126 (1987), no. 2, 439-447.

29. Ch. D. Sogge, Eigenfunctions and Bochner-Riesz estimates on manifolds with boundary, Math. Res. Lett. 9 (2002), no. 2-3, 205-216.

30. G. Szegö, Orthogonal Polynomials, 4 th ed., Amer. Math. Soc. Coll. Publ., No. 23, Providence, R.I., 1975.

31. N. Tarkhanov, The Analysis of Solutions of Elliptic Equations, Kluwer Academic Publishers, Dordrecht, NL, 1997.

Department of Mathematics and Computer Science, Bar-Ilan University, 52900 RaMAT-GAN, ISRAEL

E-mail address: aizenbrg@macs.biu.ac.il

Institute of Mathematics, University of Potsdam, PO Box 6015 53, 14415 Potsdam, Germany

E-mail address: tarkhanov@math.uni-potsdam.de 